\title{
Outcomes in patients treated with gamma knife radiosurgery for brain metastases from malignant melanoma
}

\author{
Gerhard M. Friehs, M.D., Joseph Legat, M.D., Zhen Zheng, Ph.D., Gerhard Pendl, M.D., and \\ Georg C. Noren, M.D., Ph.D. \\ Department of Clinical Neurosciences (Neurosurgery), Brown University, Providence, Rhode Island; \\ and Department of Neurosurgery, Karl-Franzens University, Graz, Austria
}

In order to determine the effectiveness of gamma knife (GK) radiosurgery in patients with malignant melanoma metastases, the authors conducted a prospective multicenter study. :Forty-five patients with a total of 96 lesions were treated and followed to measure survival time, tumor control rate, and Karnofsky Performance Scale (KPS) score. The mean survival time was 8.7 months (median 4.2 months), and tumor control was achieved in $86 \%$ of lesions. When obtained, the median preoperative KPS of 80 was maintained at a median of 80 post-GK treatment.

The authors found GK treatment for metastases of malignant melanoma to be highly effective in controlling tumor growth. Survival rates obtained after GK treatment were found to be superior to historically obtained data concerning external beam radiotherapy follow up. Radiosurgery with the GK can therefore be considered a good primary treatment option for metastatic brain disease of malignant melanoma.

Key Words * gamma knife radiosurgery * malignant melanoma * brain metastasis * outcome * survival

Malignant melanoma is one of the many cancerous tumors that metastasize to the brain. In the past the only treatment options for patients with malignant melanoma metastases were surgery and radiation therapy. Surgical removal, as for other metastatic tumors, is usually advocated in cases of singular metastatic lesions to the brain. External beam radiation therapy (XRT) has been used and studied extensively, but melanoma metastases in the brain are considered rather unresponsive to XRT and therefore "radioresistent."[4,15] The body of evidence regarding the value of focused radiation for brain metastatic lesions is growing steadily. Gamma knife (GK) and stereotactic linear accelerator radiosurgery treatments have been applied successfully for single and/or multiple lesions, either in addition to or instead of XRT.[13,14,17,18] In these numerous reports it was postulated that treatment with radiosurgery was able to overcome the radioresistence of melanoma metastases of the brain.

This report summarizes the course of 45 patients diagnosed with malignant melanoma metastatic to the 
brain. The purpose of this report was to determine the survival times after GK radiosurgery as well as the likelihood of tumor control. Our hopes were that outcomes with GK radiosurgery would at least equal historical outcomes data on XRT, and these were met.

\section{CLINICAL MATERIAL AND METHODS}

Between April 1992 and April 1998 a total of 45 patients with malignant melanoma metastatic to the brain were treated in two GK centers. Both centers are part of urban community-based hospitals that serve populations of approximately 1.5 million people each. Patients were referred for GK treatment either directly by their family physicians or dermatologists or indirectly through recommendation of family members or friends. Diagnosis was based on histopathological examination of surgically removed tumor, stereotactic biopsy, or clinical suspicion with a known primary malignant melanoma site. The mean age was 57 years (range 26-79 years); 28 patients were men and 17 were women. One to six tumors per patient were detected and a total of 96 lesions treated, with an average dose to the tumor margin of 15 to $30 \mathrm{~Gy}$ (maximum dose 16.7-75 Gy); the isodoses used to cover the tumor varied between 30 to $90 \%$. Average pretreatment tumor volumes were found to be $5 \mathrm{~cm}^{3}$ with a range between 0.03 to 25 $\mathrm{cm}^{3}$. Whenever possible, patients were seen in follow-up intervals of 6 to 12 weeks posttreatment and imaging studies were obtained within the same time frame.

Patients were followed prospectively because all patients who undergo GK radiosurgery in both institutions are automatically entered into a computer database. The point of entry into the study was the date of GK treatment. The primary outcome measure was length of survival after GK treament. Secondary measures were tumor control rate as defined by tumor size post- compared to pre-GK (smaller, unchanged, larger) and performance status was measured with the Karnofsky Performance Scale (KPS). Tumors were considered "controlled " if they decreased in size or remained the same.

\section{RESULTS}

Complete follow up after GK treatment was obtained for all 45 patients. Survival rates ranged from 0.3 months to almost 6 years with a median of 4.2 months (average 8.7 months). Table 1 provides a summary of the actual data and also gives an indication of the time between detection of the melanoma and occurence of brain metastases. Follow-up data on lesion sizes were obtained only for 71 (74\%) of 96 lesions because some follow-up images were either not obtained or not sent to the GK centers for evaluation. Of these 71 tumors, $15(21 \%)$ had decreased, $46(65 \%)$ remained unchanged, and $10(14 \%)$ had increased in size. Therefore, overall tumor control in this subpopulation was $86 \%$. Karnofsky Performance Scale scores were obtained for 35 (78\%) of 45 patients before treatment and at each postoperative follow-up visit. The median pre-GK KPS score for these patients was 80 , and remained at 80 post-GK. 


\begin{tabular}{|c|c|c|}
\hline $\begin{array}{r}\text { SUMmaR } \\
\text { D }\end{array}$ & $\begin{array}{l}\text { TABLE } 1 \\
\text { WAL DATA IN } \\
\text { ND GK RAD }\end{array}$ & $\begin{array}{l}\text { TIENTS AF TER } \\
\text { GERY }\end{array}$ \\
\hline \multirow[b]{2}{*}{$\begin{array}{l}\text { Case } \\
\text { No. }\end{array}$} & \multicolumn{2}{|c|}{ Strvival (mos) } \\
\hline & $\begin{array}{c}\text { After } \\
\text { Diagnosis }\end{array}$ & $\begin{array}{l}\text { After GK } \\
\text { Therapy }\end{array}$ \\
\hline 1 & 48 & 1.1 \\
\hline 2 & 76.5 & 3.5 \\
\hline 3 & $51^{x}+$ & 3.5 \\
\hline 4 & 15 & 1.5 \\
\hline 5 & 6 & 4 \\
\hline 6 & 28.3 & 2.3 \\
\hline 7 & $33.6 \dagger$ & 0.6 \\
\hline 8 & 173.2 & 17.2 \\
\hline 9 & 53.6 & 11.6 \\
\hline 10 & $52.4 \dagger$ & 6.4 \\
\hline 11 & $20 \dagger$ & 5 \\
\hline 12 & 147.4 & 3.4 \\
\hline 13 & 12.1 & 7.1 \\
\hline 14 & $31.6^{x}$ & 15.6 \\
\hline 15 & 99.9 & 3.9 \\
\hline 16 & 27.7 & 9.7 \\
\hline 17 & 97.7 & 1.7 \\
\hline 18 & $\ddagger$ & 4.5 \\
\hline 19 & 204 & 12 \\
\hline 20 & 31.5 & 19.5 \\
\hline 21 & 40 & 3 \\
\hline 22 & 46.3 & 0.3 \\
\hline 23 & 14.9 & 10.2 \\
\hline 24 & $18.5 \dagger$ & 2.5 \\
\hline 25 & $\ddagger$ & 9.4 \\
\hline 26 & 86.8 & 70.8 \\
\hline 27 & 71.3 & 54.4 \\
\hline 28 & $3.1 \dagger$ & 1.3 \\
\hline 29 & 56.2 & 4.8 \\
\hline 30 & 17.1 & 2.1 \\
\hline 31 & $51.3 \dagger$ & 8.5 \\
\hline 32 & $31.8 \dagger$ & 4.8 \\
\hline 33 & $44.4 \dagger$ & 20.4 \\
\hline 34 & 4.3 & 3.1 \\
\hline 35 & 28.1 & 9.1 \\
\hline 36 & 21.2 & 3.2 \\
\hline 37 & 34.2 & 5.2 \\
\hline 38 & $27.9 \dagger$ & 0.9 \\
\hline 39 & $70.1 \dagger$ & 3.1 \\
\hline 40 & 27.1 & 9.1 \\
\hline 41 & $103.3 \dagger$ & 12.3 \\
\hline 42 & $26.4 \dagger$ & 3.4 \\
\hline 43 & 6.2 & 4.2 \\
\hline 44 & 31.1 & 4.1 \\
\hline 45 & 22.3 & 9.3 \\
\hline & SK the & t failure \\
\hline
\end{tabular}

A typical response to GK treatment is seen in Fig. 1. This unfortunate 65-year-old man (Case 29) was diagnosed with malignant melanoma in his thumb, for which he underwent extensive surgery. Approximately 4 years later he developed seizures and underwent magnetic resonance (MR) imaging, which revealed his tumor (Fig. 1 left). He underwent GK radiosurgery, which resulted in significant 
improvement of his epilepsy. Although the tumor in the left motor cortex was still visible on the 3-month post-GK follow-up MR image, it had decreased in size by approximately 50\% (Fig. 1 right). The patient died less than 5 months after GK treatment from progression of his metastases outside the brain.
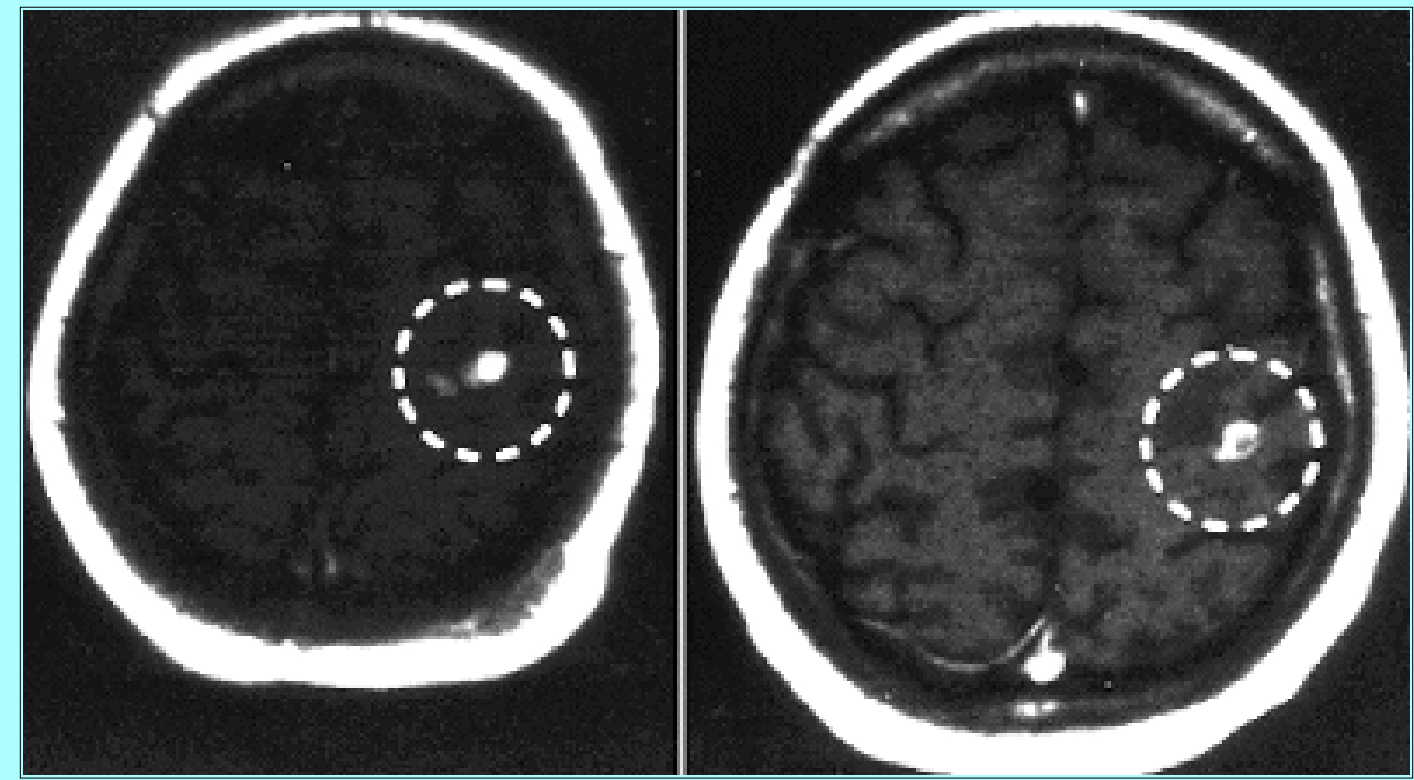

Fig. 1. Gadolinium enhanced $\mathrm{T}_{1}$-weighted $\mathrm{MR}$ images of brain metastasis from malignant melanoma in a 65 year-old male. The tumor shows clear decrease in size when comparing the pre-GK image (left) to the 3 month post-GK image (right).

Distant failure after GK treatment is shown Fig. 2. This patient (Case 3, Table 1) developed malignant melanoma on his back that metastasized to the brain. At the time of GK radiosurgery (Fig. 2 left), two lesions were found and treated. On the 3-month follow-up MR image (Fig. 2 right) the lesion in the right trigone showed clear decrease in size, whereas the other lesion in the left frontal lobe (not shown) was found to be larger and surrounded by marked edema. The patient deteriorated neurologically and became hemiparetic. He was admitted for surgical resection of the left frontal lesion which was found to be largely radiation necrosis. However, the follow-up MR image (Fig. 2 right) also depicted three new lesions for which the patient received XRT. As of completion of this study he is still alive.

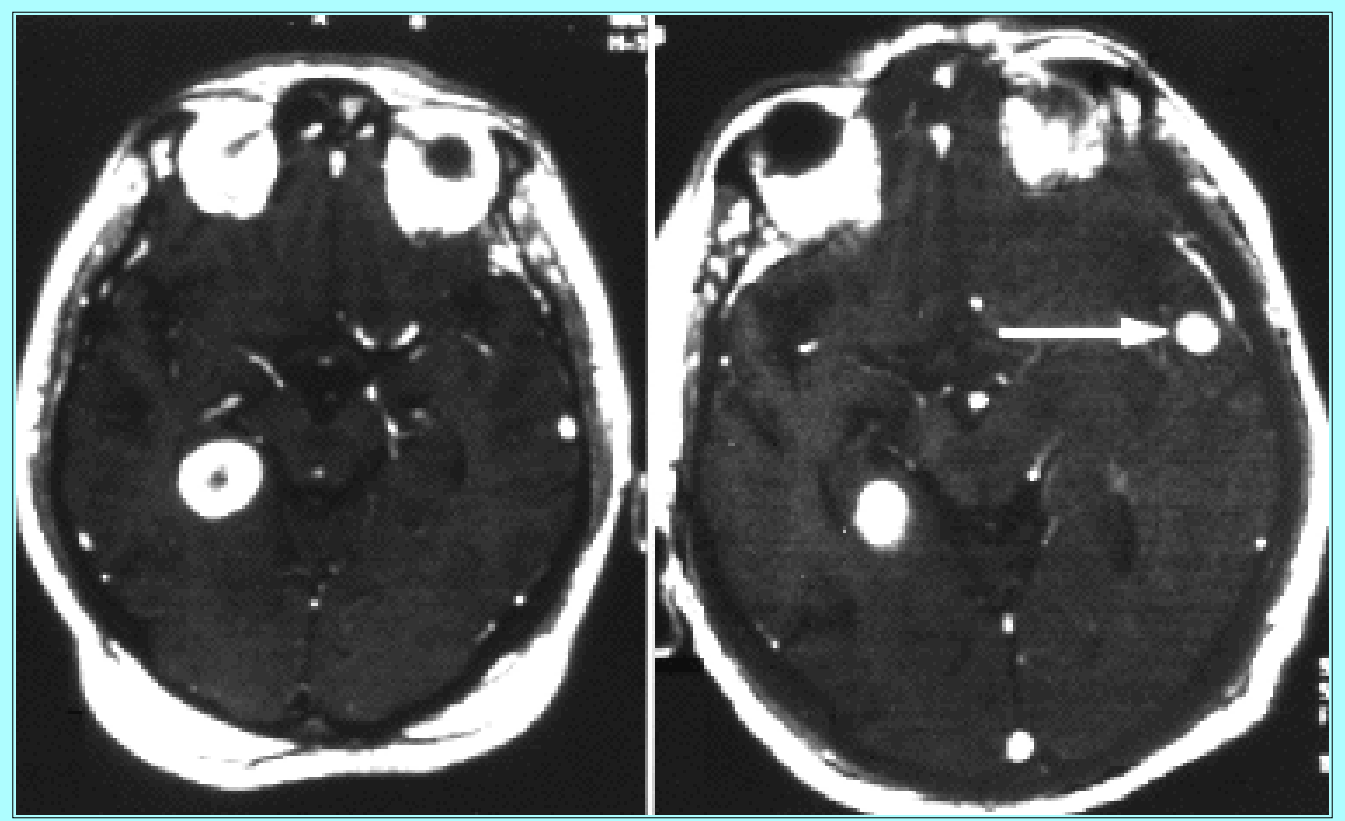


Fig. 2. Gadolinium enhanced $T_{1}$-weighted $M R$ images illustrating distant failure after GK treatment. Compared to the pretreatment MR image (left), the MR image obtained 3 months after GK treatment (right) shows the treated lesion in the right trigone, which has decreased significantly in size. However, a new lesion is detected in the left temporal region (white arrow), which was not seen 3 months prior.

\section{DISCUSSION}

Malignant melanoma, like lung and breast cancers, is one of the most common tumors metastasizing to the brain.[10,12] Once the disease has spread to the brain, survival is very short if not treated. The possible treatment options at this point include surgery, XRT, chemotherapy, and of late also radiosurgery. To our knowledge there is no report in the literature to argue convincingly that chemotherapy has a significant benefit on quality of life or survival in patients with malignant melanoma metatatic brain disease.[2] Surgery is reserved for patients with solitary, surgically accessible metastatic lesions and is usually followed by some form of adjuvant therapy.[3,6,8-11] Although XRT is often considered the "standard of care" in the treatment of metastatic brain disease, its success in treating brain metastases from malignant melanoma is overestimated. Although XRT has been reported to cause short-term symptomatic and neurological improvement,[4] the overall survival is poor and generally quoted as 2 to 4 months. Furthermore, the introduction of different fractionation schedules with higher doses per fraction has not been conclusively demonstrated to improve this dismal prognosis. $[5,16,19]$ The efficacy of stereotactic radiosurgery with linear accelerator or GK has been studied quite extensively. Average survival rates are reported to be approximately 8 months or more and therefore up to twice as long as those following XRT,[12-14] if the dose to the tumor margin is adequate.[7] The tumor control rate is found to be as high as $97 \%[14]$ and is therefore not different from metastatic tumors of other origins. $[17,18]$

When performing studies to determine the natural history of a disease or the prognosis with treatment, we are tempted to overemphasize the importance of our own findings and reject theories in conflict with our own hypotheses. To determine the ways in which our findings influence how we practice, we must ask at least the following general questions: 1) Is it reasonable to assume that the findings are reproducible and that the observed parameters did not only occur by chance? 2) Are factors other than the treatment itself potentially responsible for the good/bad outcomes? and 3) Are the findings only true for our patient population or can this population serve as an adequate sample for all patients with similar problems?

In order to allow for comparison of the data in our own study with reports from the literature and future clinical studies, we followed the clinical epidemiology guidelines that are well established and described in detail in the respective literature.[1]

In this prospective study of 45 patients treated for malignant melanoma metastases in the brain with GK radiosurgery at two centers, approximately $70 \%$ of the patients died due to progression of systemic disease and $30 \%$ had progression within the brain with local or distal failure. The mean survival rate in our series is very comparable to the reports in the literature (mean 8.7 months, median 4.2 months). We believe that this beneficial effect is largely due to GK radiosurgery, although 10 (22\%) of 45 patients received XRT to the brain at some point during their disease. 


\section{TABLE 2}

CRITICAL ANALYSS OF THS STUDV ACCORD NG TO EPIDEMOLOGICAL CRTERIA

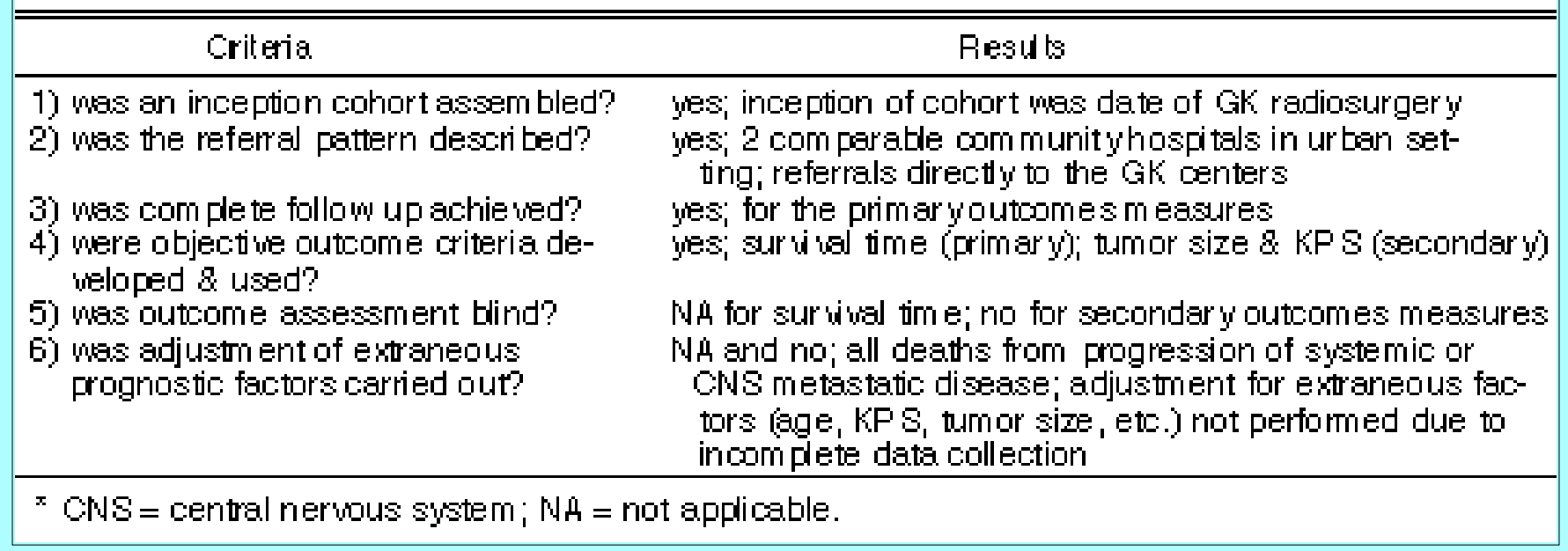

Table 2 outlines the six basic questions that are necessary to determine the epidemiological value of the study presented here. Based on this analysis we believe that the evidence generated by our data is strong enough to make GK radiosurgery for the treatment of melanoma metastasis to the brain a strong treatment option. Given these results we conclude that GK radiosurgery, if available, should be strongly considered as the primary treatment for patients with melanoma metastatic to the brain. Survival after GK radiosurgery can be expected to be at least as long as with XRT. Radiosurgery using the GK has the advantage of being a 1-day treatment as opposed to 10 or more sessions of XRT. Therefore, the time spent with treatment is significantly reduced for patients undergoing GK radiosurgery, which is especially important for patients who have only a limited amount of time to live.

\section{References}

1. Anonymous: How to read clinical journals: III. To learn the clinical course and prognosis of disease. Can Med Ass J 124:869-872, 1981

2. Brand CU, Ellwanger U, Stroebel W, et al: Prolonged survival of 2 years or longer for patients with disseminated melanoma. An analysis of related prognostic factors. Cancer 79:2345-2353, 1997

3. Brega K, Robinson WA, Winston K, et al: Surgical treatment of brain metastases in malignant melanoma. Cancer 66:2105-2110, 1990

4. Carella RJ, Gelber R, Hendrickson F, et al: Value of radiation therapy in the management of patients with cerebral metastases from malignant melanoma: Radiation Therapy Oncology Group Brain Metastases Study I and II. Cancer 45:679-683, 1980

5. Choi KN, Withers HR, Rotman M: Intracranial metastases from melanoma. Clinical features and treatment by accelerated fractionation. Cancer 56:1-9, 1985

6. Ewend MG, Carey LA, Brem H: Treatment of melanoma metastases in the brain. Semin Surg Oncol 12:429-435, 1996

7. Gieger M, Wu JK, Ling MN, et al: Response of intracranial melanoma metastases to stereotactic radiosurgery. Radiat Oncol Invest 5:72-80, 1997

8. Gupta G, Robertson AG, MacKie RM: Cerebral metastases of cutaneous melanoma. Br J Cancer 
9. Hagen NA, Cirrincione C, Thaler HT, et al: The role of radiation therapy following resection of single brain metastasis from melanoma. Neurology 40:158-160, 1990

10. Saha S, Meyer M, Krementz ET, et al: Prognostic evaluation of intracranial metastasis in malignant melanoma. Ann Surg Oncol 1:38-44, 1994

11. Salvati M, Cervoni L, Caruso R, et al: Solitary cerebral metastasis from melanoma: value of the 'en bloc' resection. Clin Neurol Neurosurg 98:12-14, 1996

12. Sampson JH, Carter JH, Friedman AH, et al: Demographics, prognosis and therapy in 702 patients with brain metastases from malignant melanoma. J Neurosurg 88:11-20, 1998

13. Seung SK, Sneed PK, McDermott MW, et al: Gamma knife radiosurgery for malignant melanoma brain metastases. Cancer J Sci Am 4:103-109, 1998

14. Somaza S, Kondziolka D, Lundsford LD, et al: Stereotactic radiosurgery for cerebral metastatic melanoma. J Neurosurg 79:661-666, 1993

15. Stridsklev IC, Hagen S, Klepp O: Radiation therapy for brain metastases from malignant melanoma. Acta Radiol Oncol 23:231-235, 1984

16. Vlock DR, Kirkwood JM, Leutzinger C, et al: High-dose fraction radiation therapy for intracranial metastases of malignant melanoma: a comparison with low-dose fraction therapy. Cancer 49:2289-2294, 1982

17. Williams J, Enger C, Wharam M, et al: Stereotactic radiosurgery for brain metastases: comparison of lung carcinoma vs. non-lung tumors. J Neurooncol 37:79-85, 1998

18. Young RF: Radiosurgery for the treatment of brain metastases. Semin Surg Oncol 14:70-78, 1998

19. Ziegler JC, Cooper JS: Brain metastases from malignant melanoma: conventional vs. high-dose-per-fraction radiotherapy. Int J Radiat Oncol Biol Phys 12:1839-1842, 1986

Manuscript received April 16, 1998.

Accepted in final form June 8, 1998.

Address reprint requests to: Gerhard M. Friehs, M.D., 2 Dudley Street, MOB 555, Providence, Rhode Island 02905.email: Gerhard_Friehs@ brown.edu. 